\title{
La política de cuerpos académicos en una universidad pública
}

Felipe Hernández Hernández*

Artículo de investigación sobre la política educativa de los cuerpos académicos como estrategia para el desarrollo de la investigación en las universidades.

Recibido: 28 de septiembre de 2020

Evaluado: 22 de enero de 2021

Aceptado: 22 de febrero de 2021

Publicado: 1 de julio de 2021

Citar como:

Hernández, F. (2021). La política de cuerpos académicos en una universidad pública.

Hallazgos, 18(36), 215-247. https://doi.org/10.15332/2422409X.6173

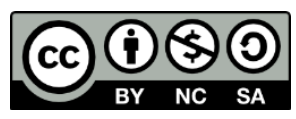

\section{Resumen}

Este artículo analiza el Programa para el Desarrollo Profesional Docente (Prodep) que impulsa la investigación en educación superior en México, con base en los grupos de investigación denominados cuerpos académicos (CA). El estudio examina el impacto de esta política educativa de formación y consolidación de CA en el periodo 1996-2019 en la Universidad Autónoma de Tlaxcala, que es de carácter estatal. El objetivo es valorar el impulso a la investigación mediante la formación de investigadores en los CA conforme a lo dispuesto por el Prodep. El enfoque metodológico es mixto. Emplea el método de análisis de

\footnotetext{
${ }^{*}$ Doctor en Ciencias Administrativas. Profesor investigador de la Facultad de Ciencias de la Educación de la Universidad Autónoma de Tlaxcala, México.

Correo electrónico: luisfel96@hotmail.com

ORCID: https://orcid.org/0000-0003-1153-1002
}

Hallazgos

ISSN: 1794-3841 | e-ISSN: 2422-409X | DOI: https://doi.org/10.15332/2422409X

Vol. 18 N.o 36| julio-diciembre del 2021 
contenido que incluyó un estudio ideológico y otro reflexivo, y un análisis de antes-después y el uso del enfoque top down de Sabatier. Los resultados muestran en el lapso de 25 años que, a pesar del tiempo que tardó el Prodep en asentarse en la comunidad académica de esta universidad, ha tenido un impacto positivo, estimulando la formación y el desarrollo de los CA y mejorando los niveles de competitividad y habilitación académica de sus profesores. Es necesaria la implementación de políticas institucionales consistentes y duraderas para que, junto con las políticas nacionales, se logre el propósito de consolidar la investigación en las universidades.

Palabras clave: Cuerpos académicos; Investigación; Política educacional; Universidad.

\section{The policy of academic bodies at a public university}

\section{Abstract}

This article makes analyzes the Program for Teacher Professional Development (PRODEP) that promotes research in higher education in Mexico, based on research groups called academic bodies. The study analyses the impact of this educational policy for the training and consolidation of academic bodies in the period 1996-2019 at the Universidad Autónoma de Tlaxcala, a public university. The objective is to assess the impetus for research through training researchers at academic bodies in accordance with the provisions of the PRODEP. The methodological approach is mixed; it uses the content analysis method that included an ideological and a reflective study, as well as a beforeafter analysis and the use of Sabatier's top-down approach. The results show that, despite the time it took for PRODEP to be accepted in the academic community of this university, it has had a positive impact, encouraging the training and development of academic bodies and improving the levels of competitiveness and academic qualifications of its professors. It is necessary to implement consistent and lasting 
institutional policies so that, together with national policies, the purpose of consolidating research in universities can be achieved.

Keywords: Academic bodies; Research; Educational policy; University.

\section{Política de corpos acadêmicos em uma universidade pública}

\section{Resumo}

Este artigo analisa o Programa de Desenvolvimento Profissional Docente (Prodep), que promove a pesquisa em educação superior no México, a partir de grupos de pesquisa denominados corpos acadêmicos (CA). O estudo analisa o impacto desta política educacional para a formação e consolidação de CA no período 1996-2019 na Universidade Autônoma de Tlaxcala, que é de natureza estadual. O objetivo é avaliar o impulso à pesquisa por meio da formação de pesquisadores nos $\mathrm{AC}$ de acordo com o que prevê o Prodep. A abordagem metodológica é mista. Emprega-se o método de análise de conteúdo que incluiu um estudo ideológico, um estudo reflexivo, uma análise antes-depois e o uso da abordagem topdown de Sabatier. Os resultados mostram ao longo de 25 anos que, apesar do tempo que o Prodep demorou a se estabelecer no meio acadêmico desta universidade, houve um impacto positivo, estimulando a formação e o desenvolvimento dos CA e melhorando os níveis de competitividade e qualificação acadêmica de seus professores. A implementação de políticas institucionais consistentes e duradouras é necessária para que, em conjunto com as políticas nacionais, se atinja o propósito de consolidação da pesquisa nas universidades.

Palavras-chave: Corpos acadêmicos (CA); Pesquisa; Política educacional; Universidade.

\section{Introducción}

La política educativa nacional en las últimas décadas ha instituido estrategias de apoyo para que en las instituciones de educación superior 
(IES) se favorezca el trabajo científico a la par del trabajo académico, es decir, hacer ciencia y no solo docencia, hacer investigación y no solo gestión. El Programa para el Desarrollo Profesional Docente (Prodep) es una de las principales estrategias en su propósito de habilitar al personal docente para realizar investigación y en su articulación en cuerpos académicos (CA).

El Prodep es una iniciativa de la Secretaría de Educación Pública (SEP) para que el personal docente de educación superior y sus CA accedan a programas de formación, actualización y capacitación o proyectos de investigación. Este programa se propuso contribuir a la profesionalización de los profesores de tiempo completo (PTC) a fin de que logren las capacidades de investigación-docencia, desarrollo tecnológico e innovación, y se articulen y consoliden en CA (Diario Oficial de la Federación [DOF], 2016), entendido como el grupo de PTC que trabaja de manera colaborativa, cuenta con la máxima habilitación académica (posgrado) y cumple con las funciones de docencia, tutoría, gestión e investigación (DOF, 2010).

Durante el tiempo que ha estado vigente este programa ha incidido en las universidades públicas, a fin de que sus académicos alcancen los niveles necesarios para desarrollar la docencia y la investigación. Sin embargo, surgen algunas preguntas al respecto: 1) ¿En qué medida los profesores han mejorado sus niveles de competitividad y habilitación académica? 2) ¿Qué situación tienen estas instituciones respecto a los CA? 3) ¿La formación y el desarrollo de los CA ha tenido un crecimiento similar en las distintas áreas del conocimiento? 4) ¿Cuáles han sido los factores internos que han influido en la integración, el desarrollo y la consolidación de los CA en las universidades?

Estos cuestionamientos inducen a la reflexión a partir de dos supuestos: primero, el Prodep representa la política educativa en educación superior 
que promueve la investigación científica, a través de la integración, el desarrollo y la consolidación de los CA; segundo, los CA son una estrategia central para la formación, el desarrollo y la consolidación de la investigación y los investigadores en la educación superior. Esto coincide con Suárez-Amaya et ál. (2021), quienes señalan que "la definición de lineamientos para la acción mediante políticas públicas, el impulso ofrecido a partir de la inversión de recursos y talento, y la existencia de indicadores que miden resultados y comparan instituciones, permiten ofrecer una visión del panorama competitivo" (p. 282) de las universidades.

El objetivo de este análisis de valorar el impulso a la investigación mediante la formación de investigadores en los CA conforme a los lineamientos establecidos por el Prodep, se realizó en una universidad pública estatal de México: la Universidad Autónoma de Tlaxcala (UATx), que al inicio de este programa (1996) tenía una planta de profesores principalmente con estudios de licenciatura y dedicada exclusivamente a la docencia. En la actualidad presenta un panorama distinto.

En este sentido, Suárez-Amaya et ál. (2021) reconocen como protagonista principal al propio investigador y refieren que, entre otros aspectos, resulta relevante conocer el número de investigadores y su crecimiento anual, el número de doctores y su incremento anual, los programas de estímulos individuales, los programas de estímulos colectivos y la creación de patentes como factores clave del éxito para el desarrollo de la investigación en las universidades.

El estudio se basó en un enfoque metodológico mixto que emplea el método de análisis de contenido en los términos que plantea Fernández (2002). Incluyó un análisis en su sentido ideológico para identificar los posibles significados que la formación y el desarrollo de los CA podrían transmitir y para develar el impacto del Prodep. Este acercamiento 
ideológico requirió un análisis cuantitativo de tipo longitudinal, de antesdespués, como propone Cardozo (2013), así como el uso del enfoque top down de Sabatier (Revuelta, 2007) para analizar el comportamiento de las personas, la valoración del logro alcanzado y la identificación de factores que pudieron influir en los resultados.

\section{El Programa para el Desarrollo Profesional Docente}

El Prodep, inicialmente Programa de Mejoramiento del Profesorado (Promep), creado en 1996 (SEP, 2006), fue resultado del trabajo conjunto entre la Asociación Nacional de Universidades e Instituciones de Educación Superior (Anuies), el Consejo Nacional de Ciencia y Tecnología (Conacyt), la Subsecretaría de Educación e Investigación Tecnológicas (SEIT) y la Subsecretaría de Educación Superior e Investigación Científica (Sesic). Este programa, sustentado en el Plan Nacional de Desarrollo 19952000 y el Programa de Desarrollo Educativo 1995-2000, se presentó en el marco de la XXVII Sesión Ordinaria de la Asamblea General de la Anuies (UAT, 2010), y se fundamentó "en las recomendaciones que la Unesco había hecho desde 1966, en ellas se menciona que el país necesitaba crear un sistema de profesionalización para que los docentes de educación superior contaran con las capacidades de investigación y docencia" (DOF, 2016, p. 15) propias para el trabajo académico en este nivel educativo. La situación que prevalecía en la educación superior en el momento de creación del Promep reflejaba que un porcentaje elevado de profesores de carrera no contaba con el nivel académico de doctorado. Tampoco estaban articulados para realizar las tareas de investigación (cuerpos académicos), y se reconocía que la calidad de la educación superior dependía de múltiples factores, pero uno de los más importantes es el profesor de carrera, quien debía contar con una formación completa para poder realizar con calidad sus funciones académicas (SEP, 2006). 
Los objetivos que estableció en sus inicios fueron:

Mejorar el nivel de habilitación del personal académico de tiempo completo en activo de las instituciones públicas de educación superior (vertiente individual) y fomentar el desarrollo y consolidación de los CA adscritos a las dependencias de educación superior de esas instituciones (vertiente colectiva). (SEP, 2006, p. ix)

La vertiente individual se caracterizaba por apoyar a las IES con becas para que sus profesores realizaran estudios de posgrado, la contratación de nuevos PTC y la entrega de un reconocimiento denominado perfil deseable, que significa que el profesor cuenta con la habilitación requerida para el desempeño docente en el nivel superior, mientras que la vertiente colectiva se orientaba a la formación de CA y al establecimiento de redes de investigación entre académicos y CA.

El Promep estuvo vigente hasta 2013. Desde el año siguiente lo sustituye el Prodep, lo articula con acciones hacia los demás niveles educativos y retoma los apoyos y reconocimientos del Promep para el tipo superior. En esta nueva etapa establece como su objetivo general "contribuir al desarrollo profesional de los docentes y cuerpos académicos de las instituciones públicas de educación superior, mediante la habilitación académica y la investigación" (DOF, 2013, p. 27).

Respecto a la investigación, señaló como su objetivo específico "profesionalizar a los PTC de las instituciones públicas de educación superior para que estos alcancen las capacidades en investigacióndocencia y se articulen y consoliden en cuerpos académicos" (DOF, 2013, p. 28). Si bien mantiene las vertientes individual y colectiva, estableció algunas particularidades para cada una de ellas. En cuanto a la primera, se orientó al reconocimiento y el apoyo a PTC con perfil deseable, el apoyo a la reincorporación de exbecarios Promep, el apoyo a la incorporación de 
nuevos PTC y el otorgamiento de becas para estudios de posgrado de alta calidad. Mientras que para la segunda dirigió su apoyo al fortalecimiento de los CA, la integración de redes temáticas de colaboración de CA, los gastos de publicación, el registro de patentes y las becas posdoctorales. Este programa tenía establecido originalmente un plazo de diez años para alcanzar sus objetivos (SEP, 2006; Edel-Navarro et ál., 2018). Sin embargo, aun con los cambios que ha habido en la administración federal, ha seguido vigente y ha mantenido prácticamente intacto su objetivo esencial de que los académicos de tiempo completo se dediquen a la docencia y también a la investigación científica.

El Prodep estaba inicialmente dirigido a las universidades públicas estatales (UPES), al Instituto Politécnico Nacional (IPN) y al Instituto Tecnológico de Sonora (Itson), pero paulatinamente se fueron adhiriendo otras modalidades de educación superior, como las universidades politécnicas, tecnológicas, interculturales, institutos tecnológicos, incluso, las escuelas normales, por lo que de 35 IES que tenía en 1996, para 2016 ya sumaban 730 (Edel-Navarro et ál., 2018). Algunos aspectos de esta política pública que han estado en la mesa de la discusión por los académicos y las autoridades universitarias son su viabilidad, sus efectos en la organización de las universidades y su impacto en la mejora de la calidad de la educación, entre otros.

Desde los años ochenta, y con mayor consistencia a partir de la década de los noventa, se establecieron distintas acciones para orientar la política educativa que evalúa la educación superior. Esta se realiza en cuatro niveles: la institución, los programas, los estudiantes y los académicos. Entre estas acciones están el Programa Nacional de Modernización Educativa (PNME), la Comisión Nacional para la Evaluación de la Educación Superior (Conaeva), el Sistema Nacional de Evaluación de la Educación (SNEE) y los Comités Interinstitucionales para la Evaluación de 
la Educación Superior (Ciees), así como otras más focalizadas a la evaluación del desempeño académico, como el Sistema Nacional de Investigadores (SNI), los programas de estímulos a la carrera docente y el Promep (Magaña, 2016).

Respecto al fortalecimiento de la investigación, la innovación y la tecnología en las IES, sobresalen el SNI y el Prodep, aunque si bien el primero valora especialmente el trabajo individual y el segundo el trabajo colegiado, entre ambos generan una tensión en los académicos y las instituciones, ya que, como menciona Magaña (2016), citando a De Sierra, existe una articulación entre estas instancias que los hace operar como un sistema, dados los condicionamientos recíprocos que establecen para pertenecer a estos.

La factibilidad e idoneidad del Prodep se ha cuestionado desde sus inicios. Mencionan Edel-Navarro et ál. (2018), por ejemplo, la insuficiencia presupuestal para apoyar la superación académica. En 2002 este programa tenía un presupuesto de 301.3 millones de pesos para atender a 70 instituciones, mientras que para 2016, con un presupuesto de 825.1 millones debía atender a 730 IES, es decir, el presupuesto se incrementó en un $173 \%$, pero el número de instituciones creció en un $943 \%$. Esto significa que en 2002 cada institución en promedio podía contar con alrededor de 4300000 pesos mexicanos, mientras que para 2016 solo podrían disponer de un poco más de un 1100 ooo pesos mexicanos. La distribución presupuestal impacta en las diversas líneas que atiende el Promep, por lo que, aunque sus datos indican un crecimiento sustancial en la cantidad de perfiles deseables y de CA, muchos de los avances que reporta como suyos en realidad no lo son. Tal es el caso de los doctores graduados después de 1996, quienes en una gran mayoría realizaron sus estudios sin apoyo del Promep (Edel-Navarro et ál., 2018). 
Otro aspecto relevante que cuestionan es el modelo de profesor que sustenta este programa, ya que se orienta a que el académico, como profesor de tiempo completo y con estudios doctorales, realice investigación. Esta se mide por una productividad reflejada en artículos publicados en revistas científicas. Sin embargo, como está ligada a evaluaciones y estímulos económicos, genera en los profesores una distorsión del trabajo académico, pues hace que se preocupen por la búsqueda de puntajes, lo que en consecuencia los lleva a desatender la docencia (Edel-Navarro et ál., 2018). Desde la perspectiva de los programas merit pay, los profesores realizan todo aquello que es objeto de un puntaje en la evaluación, y por ello dejan de lado las tareas sustantivas de la responsabilidad docente (Díaz-Barriga, 2005).

Dada la complejidad que significa ingresar al SNI, no así la obtención del perfil deseable Prodep, aunado a que en algunas instituciones existe falta de reconocimiento a los académicos que realizan investigación, motiva a que estos se inclinen más por cumplir los requisitos de permanencia en la docencia, por conservar el empleo, antes que por satisfacer sus funciones como docentes investigadores, aun cuando los mismos académicos reconocen ampliamente que tener este perfil les despierta la motivación, pero también les crea distanciamiento y envidias (Pérez y Bañuelos, 2017). En términos generales, el Prodep ha tenido efectos positivos en la revitalización de la planta académica, en una mayor actualización y profesionalización de los docentes, en el fomento a la investigación y a la docencia, en el incremento de redes epistémicas y la posibilidad de dedicarse de tiempo completo a la vida académica en la universidad. No obstante, también ha tenido efectos negativos: una orientación compulsiva de las instituciones al cumplimiento de sus indicadores, la deshomologación de la remuneración que lleva a una desigualdad entre los docentes y a una cada vez mayor estratificación entre los académicos y 
entre las universidades, diferentes criterios de evaluación interna, simulación en la investigación, pérdida de autonomía de las universidades, ambiente de competencia muchas veces desleal entre los investigadores, pérdida del sentido de pertenencia a los grupos y compromisos con la institución, entre otros (Magaña, 2016).

\section{Los cuerpos académicos como política educativa}

Una política pública se entiende como el conjunto de

[...] principios establecidos, generalmente, por acción legislativa y que dirigen el accionar del gobierno, especialmente de los diversos estamentos que componen su rama ejecutiva/administrativa, para tratar con un grupo de problemas establecidos, tanto en el corto como en el largo plazo. (Avendaño et ál. 2017, p. 3)

También se refiere a las acciones gubernamentales con "objetivos de interés público que surgen de decisiones sustentadas en un proceso de diagnóstico y análisis de factibilidad, para la atención efectiva de problemas públicos específicos, en donde participa la ciudadanía en la definición de problemas y soluciones” (Franco, 2017, p. 86).

Ambas definiciones coinciden en que las políticas públicas atienden problemas de interés colectivo o general, es decir, necesidades sociales, y su ejecución corresponde al mismo gobierno o a sus dependencias (García, 2019). Cabe señalar que este accionar del Gobierno puede llegar a confundirse con otras acciones específicas: políticas de Estado y políticas de Gobierno. Sin embargo, son diferentes. Las primeras se refieren a las acciones planteadas en la constitución, con preceptos claros que trascienden los periodos de gestión del Gobierno, mientras que las segundas son las acciones cotidianas que se instrumentan, según la competencia administrativa de cada una de las dependencias y entidades públicas (Franco, 2017). 
Referidas a la educación, las políticas públicas se entienden como "el conjunto de preceptos impuestos por un estado en calidad de principios rectores del accionar del sector educativo dentro de su territorio con la participación de los actores educativos para responder a los intereses públicos de la sociedad civil" (Avendaño et ál., 2017, p. 6). Tienen que ver con los planteamientos que hace el Estado para la educación, los cuales dependen del contexto, los requerimientos y la presión de los actores sociales de cada país.

Las políticas públicas tienen dos etapas importantes después de que estas se han formulado. Una es la etapa de implementación, es decir, de llevarla a la práctica, de ejecutarla, que no es otra cosa más que la puesta en marcha del plan de acción definido, lo cual incluye presupuesto, formalización legal, entrenamiento de quienes la llevarán a cabo y comunicación con los entes involucrados (Franco, 2017). Esta fase es fundamental porque se ha creído que una vez tomada la decisión, esta sería implementada, con lo que lograría los resultados similares a los que inicialmente se habían señalado. La implementación es el proceso que ocurre entre las declaraciones formales de la política y el resultado final alcanzado (Revuelta, 2007).

La otra etapa es la evaluación de impacto, es decir, valorar sus efectos para determinar cómo ha cambiado una situación una vez que ha culminado la acción del Gobierno. De algún modo es la respuesta a las preguntas "¿la política pública generó los efectos deseados?, ¿cuáles son las áreas de oportunidad para mejorar el diseño de la política pública?” (Franco, 2017, p. 93). Sin embargo, dado que la implementación es un proceso cambiante, se deben analizar las políticas públicas por periodos de por lo menos diez años o más (Sabatier citado por Revuelta, 2007).

Una particularidad que tienen las políticas públicas en educación superior en México es la continuidad que han tenido desde la década de los 
ochenta, sin que los cambios de Gobierno hayan traído rupturas o modificaciones sustanciales. De tal manera que estas se han centrado en temas específicos como la cobertura, la diversificación, la descentralización, la equidad, la pertinencia, la coordinación, la planeación y la evaluación (Avendaño et ál., 2017).

Esto se observa con claridad en la política de formación y desarrollo de los CA, que ha estado vigente por más de veinte años. Durante todo este periodo ha mantenido prácticamente incólume su objetivo de fortalecer la preparación profesional de los profesores de educación superior para que cuenten con estudios de posgrado y adquirieran la habilitación académica necesaria que les permita asegurar un desempeño de calidad en las actividades sustantivas de docencia e investigación que les corresponden realizar en este nivel educativo.

El Prodep define que un CA es un grupo de profesores de tiempo completo que tiene definidas sus líneas de generación o aplicación del conocimiento en temas disciplinares o multidisciplinares. Comparten objetivos y metas académicas; cuentan con reconocimiento de perfil deseable y con la máxima habilitación académica; tienen amplia experiencia docente; demuestran una actividad académica intensa; cuentan con productos derivados de sus actividades de investigación y participan en redes de intercambio académico con sus pares. En suma, realizan las funciones de docencia, tutoría, gestión e investigación y se clasifican según su nivel de habilitación como CA en formación (CAEF), CA en consolidación (CAEC) y CA consolidados (CAC). Cada nivel demanda el cumplimiento de determinados criterios de valoración (DOF, 2010).

Los CA, como cualquier otra organización, se forman para que los individuos puedan alcanzar fines que se entiende que por sí solos no los pueden obtener. Constituyen una unidad que consta de al menos dos personas que coordinan sus acciones para la obtención de metas comunes 
(Gibson et ál., 2006; Jones, 2008; Daft, 2004), y como unidad trabajan en grupos, utilizan conocimientos y técnicas específicas y mantienen un conjunto de relaciones estructuradas (Kast y Rosenzweig, 2004).

La integración de los académicos en CA lleva en el fondo el objetivo de que la investigación científica no sea una actividad individual sino colectiva, que no se realice de manera aislada sino en forma colaborativa. De modo que el término grupo lleva implícito el carácter de colectividad, en el que la interacción es un proceso fundamental.

Algunos de los análisis sobre esta política educativa (Rueda, 2003; Lobato y Garza, 2009) resaltan que la integración de los CA facilita la generación de conocimiento de forma colectiva, el desarrollo de proyectos de investigación, de eventos académicos, y la sistematización de información. Se distinguen por tener un conjunto de objetivos y metas comunes para realizar investigación, docencia y difusión del conocimiento. Esto les ha servido como base para escalar en la colegialidad y el trabajo en equipo. Por su parte, Zúñiga et ál. (2016) recalcan que los CA se crearon para fortalecer actividades académicas sustentadas en el trabajo colaborativo y crear modos colectivos de producción del conocimiento. Desarrollan relaciones interorganizacionales que sostienen el flujo de conocimiento desde una perspectiva específica con diferentes formas de expresión, como la formación de redes de investigación, que le dan al desarrollo de la investigación colectiva un carácter más operativo.

En el trabajo de los CA prevalece la cooperación. Esta se caracteriza por la definición de una agenda común y su participación en redes de relaciones informales y formales. Su cohesión se da por el conjunto de normas y creencias producto de su afinidad más que de lazos familiares, políticos o institucionales. Comparten un sistema de creencias y valores que definen propósitos comunes. En virtud de ser relativamente pequeñas, tienen una 
estructura compacta. Destacan el logro del prestigio académico y el fortalecimiento de su habilidad para influir en el campo de su disciplina. En su integración predominan más las relaciones informales que las formales, y su capital más importante son su reputación y sus méritos académicos (Maldonado, 2005).

Mientras que para Jornet et ál. (2012), el trabajo colaborativo es abordado "como una estrategia positiva para conseguir mejores resultados en los centros educativos, destacando sus ventajas y por otro lado como una opción política de inhibición de los docentes que responde a intereses económicos" (p. 181), para Hargreaves (1996), la idea de colegialidad en un mundo posmoderno implica concebir la solución de problemas como una pieza angular de las instituciones. Aquí se resaltan los esfuerzos para construir culturas de colaboración y desterrar las posturas del aislamiento del académico, y así ir más allá de las aportaciones individualistas. Por su parte, Becerril et ál. (2019) destacan diversos factores que influyen en la articulación de los CA, tales como la relación interpersonal y los recursos financieros disponibles; la socialización de la información producto de las investigaciones realizadas; la sinergia del CA en relación con la toma de decisiones entre los integrantes y el líder; la conducta de los investigadores, que también está determinada por los incentivos que reciben, y, especialmente, por su significado como indicador de la calidad de la educación en las IES.

En este sentido, se observa al CA como un proyecto estratégico que permite aprovechar el ámbito institucional en donde se encuentran los académicos, pero sin perder de vista sus intereses personales y compromisos con la realidad educativa y escolar (Cabrera y Díaz-Ordaz, 2013), dado que funcionan como una comunidad dual en la que un académico pertenece a una institución y también a un campo profesional o disciplina (Clark, 1998). 
La comunidad académica es un elemento inherente tanto en la definición como en la operatividad de los CA, ya que no existe una sola comunidad, sino muchas, y a diferentes niveles.

Casi todos los investigadores asumen una filiación comunitaria, dan por supuesto la responsabilidad de llevar a cabo las tareas que corresponden a las diferentes especialidades de la disciplina, y se distribuyen entre grupos cuya membresía está más o menos determinada. (Olivé, 2008, p. 30)

Entre las múltiples creencias en que se ha basado la formación de los CA está la idea de que

[...] los académicos, independientemente de las disciplinas, contextos institucionales, tradiciones, creencias y hábitos de trabajo, tienden a agruparse para producir conocimiento, transmitirlo o difundirlo. Y aquí, como ocurre con todas las creencias, hay problemas. No todos los académicos en todas las disciplinas actúan así, ni todo el tiempo. Más bien, si uno lo mira sobre todo en disciplinas humanísticas y en algunas científicas, los académicos son tremendamente individualistas, celosos de su libertad e independencia, competitivos, solitarios. Hay factores como la edad, el género, la formación previa, los ambientes institucionales, las relaciones con sus colegas, las subdisciplinas específicas, las condiciones laborales, y otros, que inciden en el tipo de disposiciones para trabajar solos o en equipo. (Acosta, 2006, pp. 86-87)

Otro conflicto que se observa es la política educativa que estimula la individualización del trabajo académico. Aquí el acceso al SNI, a los programas de estímulos de las universidades y a la obtención del perfil Prodep está asociado a la productividad y la calificación individual. De este modo, los académicos siguen produciendo de manera individual y la integración de los CA es un hecho puramente organizacional e impuesto por la SEP (Acosta, 2006). 
Esta realidad la matizan Brambila et ál. (2016) cuando mencionan que "al publicar hallazgos en artículos de dos o más autores, los científicos dan visibilidad al grupo como parte de la comunidad disciplinar. Ser autor de un artículo publicado evidencia un reconocimiento y alimenta el posicionamiento disciplinar de cada investigador" (p. 5). Es decir, el individuo trabaja en grupo, pero también importa el reconocimiento individual como experto. La creciente complejidad de la ciencia y su gestión vuelven imperativo colaborar, pero también que cada investigador sea reconocido por sus aportaciones.

En suma, los estímulos, en lugar de unir a los CA, los fragmenta, desplazando los propósitos de la institución para ubicar a la remuneración como centro de su preocupación. Esto propicia una colaboración ficticia basada en indicadores que poco corresponden con la práctica, dado que el recurso es restringido y la evaluación individual. La participación en los CA se refleja en sus condiciones de trabajo, las cuales, inicialmente, si bien son favorables, después se convierten en una exigencia. Reconocen que la misma dinámica los lleva a producir más rápido para elevar su productividad en cantidad, pero a veces queda en duda su calidad (Pérez y Bañuelos, 2017).

La política educativa de los CA, en este sentido, se orienta a fomentar el trabajo colectivo, pero también a reconocer y estimular la producción individual. Estos dos aspectos, que al inicio se reconocen como complementarios, también pueden llegar a ser contrapuestos, lo cual crea en los investigadores un dilema en el establecimiento de sus prioridades y multiplica las demandas que deben atender.

\section{Metodología}

El enfoque metodológico de este estudio para valorar el impulso a la investigación mediante la formación de investigadores en los CA, 
conforme lo establece el Prodep, fue de corte mixto. Se utilizó el método de análisis de contenido en los términos que plantea Fernández (2002). La institución objeto de estudio fue la Universidad Autónoma de Tlaxcala. Se revisaron documentos institucionales que dan cuenta de la situación de sus CA y de sus investigadores. Después se realizó un análisis en su sentido ideológico para identificar los posibles significados que la formación y el desarrollo de los CA podrían transmitir para describir tendencias y develar el impacto de esta política pública.

El estudio en un sentido ideológico (Fernández, 2002) requirió un análisis cuantitativo de tipo longitudinal de antes-después (Cardozo, 2013). Para ello se establecieron los siguientes periodos de corte: 1996 (inicio del programa), 2002 (la Universidad registra sus primeros CA), 2009 (primer impacto del Prodep en la universidad) y 2019 (estado actual de los CA). Esta perspectiva metodológica permitió extraer la información necesaria para comprobar en qué medida el programa se acerca o no a las metas propuestas en la forma más objetiva y verificable posible (Cardozo, 2013).

Cabe resaltar también el uso del enfoque top down de Sabatier, quien estableció que este "comienza con el análisis de la toma de decisiones y después examina el grado en que sus objetivos, legalmente ordenados, fueron alcanzados en el tiempo y por qué" (Revuelta, 2007, p. 145). El uso de este enfoque incluyó el análisis del comportamiento, las personas y los grupos participantes, la valoración del logro de los objetivos establecidos y la definición de los factores que influyeron en el logro de los resultados esperados. 


\section{Análisis y discusión}

\section{La competitividad y la habilitación académica en la UATx}

La Universidad Autónoma de Tlaxcala actualmente se ubica en su entidad como la institución de educación superior con el mayor número de investigadores y de producción científica. La investigación científica en esta universidad inició en 1976 - fecha de su fundación-, cuando se propuso organizar a los maestros en comunidades de investigadores especialistas. Sin embargo, esta intención ha estado limitada por la carencia de investigadores, la separación de la investigación de las aulas, la falta de vinculación entre los niveles de licenciatura y posgrado y la insuficiencia de recursos institucionales para el financiamiento de proyectos. Por otro lado, la investigación solo se desarrollaba en los centros de investigación y en los posgrados, y los académicos trabajaban casi individualmente, como lo han reconocido en sus distintos planes de desarrollo institucional (Viñas, 2017).

Aun cuando el Prodep señala que la habilitación académica de los profesores es una vertiente distinta a los CA, existe entre ellos una estrecha relación, ya que para ir mejorando su nivel como CA sus integrantes deben contar con el Reconocimiento Prodep de Perfil Deseable (vertiente individual). Este se obtiene con el cumplimiento de las funciones de docencia, la generación o aplicación innovadora de conocimientos, las tutorías y la gestión académica-vinculación, pero sobresale la acreditación de estudios de posgrado (maestría y preferentemente doctorado). Respecto a este último criterio, el Promep señala que en 1996, a escala nacional, "de los 18.093 PTC adscritos a las universidades públicas estatales (UPE) solo el $8 \%$ contaba con doctorado y $32 \%$ tenía una especialidad o una maestría; el 60 \% restante poseía título de licenciatura" (SEP, 2006, p. 2). En el caso de la UATx, esta contaba en ese mismo año, 
al inicio del programa, fundamentalmente con una plantilla académica con estudios de licenciatura (89.3 \%) y un porcentaje mínimo (9 \%) poseía estudios de posgrado. Para 2005 a escala nacional se observaba un incremento en la capacidad académica, los profesores con estudios de doctorado llegan al $22.1 \%$; los de maestría, al $49.4 \%$, y los de licenciatura disminuyen a 26.9 \% (SEP, 2006). En esta universidad se observa también una mejora en estos mismos indicadores, los profesores con posgrado llegan al 38.1 \% y los de licenciatura disminuyen al 54.2 \%. Es hasta 2019 en que casi alcanza los indicadores nacionales de 2005, con lo que logró una plantilla de PTC que se distingue por contar con estudios de posgrado (66.3 \%), mientras que los de licenciatura representan solo el $33.7 \%$ (tabla 1).

Tabla 1. Grados académicos de docentes de la UATx por corte

\begin{tabular}{|l|l|l|l|l|l|l|}
\hline & $\begin{array}{c}\text { Sin datos } \\
(\mathbf{\% )}\end{array}$ & $\begin{array}{c}\text { Técnico } \\
\mathbf{( \% )}\end{array}$ & $\begin{array}{c}\text { Licenciatura } \\
\mathbf{( \% )}\end{array}$ & $\begin{array}{c}\text { Maestría } \\
\mathbf{( \% )}\end{array}$ & $\begin{array}{c}\text { Doctorado } \\
\mathbf{( \% )}\end{array}$ & $\begin{array}{c}\text { Totales } \\
(\mathbf{\% )}\end{array}$ \\
\hline $\mathbf{1 9 9 6}$ & & 1.7 & 89.3 & 8.2 & 0.8 & 992 \\
\hline $\mathbf{2 0 0 5}$ & 4,7 & 3 & 54.2 & 30.5 & 7.6 & 1165 \\
\hline $\mathbf{2 0 1 9}$ & & & 33.7 & 37.8 & 28.5 & 744 \\
\hline
\end{tabular}

Fuente: elaboración propia a partir de UAT (1996, 2006 y 2019a).

Nota: los datos de 1996 y 2005 incluye a PTC y profesores de tiempo parcial, mientras que 2019, exclusivamente a docentes con categoría PTC.

En este punto se observa el cumplimiento del objetivo del Promep de que las universidades incrementaran su capacidad académica, es decir, de que tengan más profesores con posgrado, preferentemente con doctorado, y menos con solo estudios de licenciatura. Esto significa que los docentes están habilitados para desarrollar las funciones propias de un profesor de nivel superior. En el caso de la UATx, es visible este hecho. Paulatinamente fueron incrementando el número de profesores con posgrado, $9 \%$ en 1996, $38.1 \%$ en 2005 y $66.3 \%$ en 2019. 
La habilitación de los profesores se observa también en el número de PTC que tienen estudios de posgrado (66.3\%), en los que cuentan con grado doctoral (28.5 \%), los que están integrados a algún CA (31.5 \%), los que tienen el reconocimiento de perfil Prodep (33.4 \%) y los miembros del SNI (12.7 \%) (tabla 2). Conforme a los objetivos del Prodep, los profesores de nivel superior deben estar habilitados para desarrollar funciones de investigación y no solo de docencia, y esto es posible cuando tienen estudios de posgrado y se incorporan a los grupos de investigación CA. En el caso de esta universidad se observa el cumplimiento de tal objetivo. Es decir, para 2019 más del 66 \% de los profesores cuentan con estudios de posgrado (maestría y/o doctorado); quienes cuentan con grado de doctor representan casi una tercera parte de la planta académica, forman parte de los CA y tienen el reconocimiento de perfil deseable Prodep. Sin embargo, los investigadores reconocidos por el SNI muestran todavía un porcentaje menor.

Tabla 2. Características de los PTC (Posgrado, CA, PRODEP y SNI)

\begin{tabular}{|l|l|l|l|l|l|}
\multicolumn{1}{c}{$\begin{array}{c}\text { Total de } \\
\text { PTC }\end{array}$} & $\begin{array}{c}\text { PTC con } \\
\text { posgrado }\end{array}$ & $\begin{array}{c}\text { PTC con } \\
\text { doctorado }\end{array}$ & PTC en CA & $\begin{array}{c}\text { Con perfil } \\
\text { PRODEP }\end{array}$ & En el SNI \\
\hline $\mathbf{7 4 4}$ & 493 & 212 & 235 & 249 & 95 \\
\hline Porcentaje & 66.3 & 28.5 & 31.5 & 33.4 & 12.7 \\
\hline
\end{tabular}

Fuente: elaboración propia a partir de UAT (2019a y 2019b).

En cuanto a los académicos reconocidos como investigadores, la UATx no tiene esta categoría en su normatividad, pero reconoce como tales a los académicos que obtienen esa distinción del SNI. Este sistema establece tres categorías: Candidato a Investigador Nacional, Investigador Nacional con tres niveles (I, II y III) e Investigador Nacional Emérito, que se otorgan en razón de la trayectoria, la productividad y la trascendencia de sus aportaciones. La UATx en 2002 incorporó sus primeros investigadores al SNI. Estos se ubicaban en las divisiones de Ciencias Sociales y 
Administrativas y de Ciencias Biológicas. En 2005 ya cuenta con 25 investigadores, 17 de los cuales tienen el nivel I y los ocho restantes ostentan la categoría de Candidatos. Para 2019 se refleja un avance considerable con 95 académicos de exclusividad, de los cuales 24 son candidatos, 59 están en el nivel I y 12 en el nivel II; no cuenta con académicos investigadores en el nivel III y tampoco eméritos (tabla 3).

Tabla 3. Académicos de la UATx integrantes del SNI

\begin{tabular}{|c|c|c|c|c|c|c|}
\hline & \multicolumn{3}{|c|}{ Categorías } & \multirow[b]{2}{*}{ Nivel III } & \multirow[b]{2}{*}{ Eméritos } & \multirow[t]{2}{*}{ Totales } \\
\hline & Candidatos & Nivel I & Nivel II & & & \\
\hline 2005 & 8 & 17 & - & - & - & 25 \\
\hline 2019 & 24 & 59 & 12 & - & - & 95 \\
\hline
\end{tabular}

Fuente: elaboración propia a partir de UAT (2006 y 2019b).

Datos del Programa de Fortalecimiento de la Calidad Educativa 2016-2017 señalan que el número de investigadores tiene un crecimiento mesurado debido a los niveles salariales, que están por debajo de los otorgados por otras IES. Por eso se han implementado estrategias diversas como incentivar el trabajo colegiado de las publicaciones, coediciones, participación en foros y espacios académicos, así como programas de estímulos como el de Trayectoria y Renivelación de Categorías Académicas y Reconocimiento del Trabajo Universitario, el Programa de Estímulos al Desempeño Docente (Esdeped) y el Programa Estratégico para Cuerpos Académicos, Calidad y Productividad para su Internacionalización (CACyPI) (UAT, 2016).

\section{El Prodep y los cuerpos académicos en la UATx}

Otro de los objetivos esenciales del Prodep, especialmente en la vertiente colectiva, es la conformación de grupos de investigación que denomina CA. Estos se organizan con el propósito de que los académicos se dediquen a la investigación y no solo a la docencia. Los CA se clasifican en distintos 
niveles en razón de su productividad y dinámica de trabajo. Si bien el programa inicia a finales de 1996, la UATx registra formalmente la aplicación de esta política educativa hasta 2002, con el registro de $10 \mathrm{CA}$ y 5 grupos disciplinarios (GD) (figura que más tarde desapareció). Para 2009, esta política ya se había asentado en la comunidad universitaria y contaba con $41 \mathrm{CA}$, de los cuales 28 estaban reconocidos como CAEF; 10, como CAEC, y 3, como CAC. En 2019 tiene registrados 57 CA, de los cuales 14 son CAEF; 21 son CAEC, y 22 están como CAC, que representan el $24.5 \%$, el $36.8 \%$ y el $38.5 \%$ respectivamente (tabla 4 ).

Tabla 4. Cuerpos académicos de la UATx por periodo de corte

\begin{tabular}{|c|c|c|c|c|c|}
\hline Periodo & CAEF & CAEC & CAC & Totales & Observaciones \\
\hline 1996 & - & - & - & - & Inicia el Prodep \\
\hline 2002 & 10 & - & - & 10 & \\
\hline 2009 & 28 & 10 & 3 & 41 & \\
\hline 2019 & 14 & 21 & 22 & 57 & \\
\hline
\end{tabular}

Fuente: elaboración propia a partir de UAT (2010) y Prodep (2019).

Este trayecto muestra cómo los académicos de esta universidad paulatinamente se fueron organizando en CA, desarrollando investigaciones, publicando sus productos y escalando en los distintos niveles que tiene establecido el programa. Es decir, en estos grupos de trabajo se hace visible el desarrollo de la investigación y la formación de investigadores. En modo alguno reflejan la habilitación docente y la dedicación a la investigación.

Si bien en términos generales estos datos muestran que este programa ha tenido un desarrollo aceptable, dado que en el periodo global de veintitrés años de existencia se ha asentado en la dinámica institucional, un análisis dentro de esta por áreas del conocimiento muestra los resultados que se presentan a continuación. En 2002, de las cuatro divisiones del conocimiento en que está organizada esta universidad, tres iniciaron con 
un número similar de CA, a excepción de la de Ciencias y Humanidades, que no registró $\mathrm{CA}$ alguno, aunque, por otro lado, fue esta área la que registró el número mayor de GD (tabla 5).

Tabla 5. CA de la UATx por división académica en 2002

\begin{tabular}{|l|l|l|}
\hline \multicolumn{1}{|c|}{ División académica } & $\begin{array}{c}\text { Cuerpos } \\
\text { académicos (CA) }\end{array}$ & $\begin{array}{c}\text { Grupos } \\
\text { disciplinarios (GD) }\end{array}$ \\
\hline Ciencias Básicas, Ingeniería y Tecnología & 4 & 0 \\
\hline Ciencias Biológicas y de la Salud & 3 & $\mathbf{1}$ \\
\hline Ciencias Sociales y Administrativas & 3 & $\mathbf{1}$ \\
\hline Ciencias y Humanidades & & 3 \\
\hline Totales & 10 & 5 \\
\hline
\end{tabular}

Fuente: elaboración propia con datos de UAT (2010).

Para 2009 (tabla 6), las divisiones de Ciencias Sociales y Administrativas y de Ciencias Biológicas y de la Salud tenían registrados el mayor número de CA, ubicando a diez de ellos como CAEC o como CAC. La de Humanidades ya tiene registrados cuatro $\mathrm{CA}$, pero todos están como CAEF. Esto último tal vez se explica por lo tardío de su aceptación o incorporación a esta política educativa.

En 2019, la distribución de CA por división se mantiene casi de manera idéntica. Las de Ciencias Sociales y Administrativas y de Ciencias Biológicas y de la Salud registran el mayor número de CA (19 y 18 respectivamente), incluso la mayoría de ellos están en el nivel de CAC; mientras que las divisiones de Ciencias Básicas, Ingeniería y Tecnología y la de Humanidades si bien muestran un crecimiento importante (10 cada uno), la mayoría de los de la primera área se ubican como CAEC, y los de la segunda están como CAEF (tabla 6). 
Tabla 6. CA de la UATx por división académica y grado de consolidación en 2009 y 2019

\begin{tabular}{|c|c|c|c|c|c|c|c|c|}
\hline \multirow{3}{*}{$\begin{array}{l}\text { Dependencia o } \\
\text { división }\end{array}$} & \multicolumn{6}{|c|}{ Categoría } & \multirow{2}{*}{\multicolumn{2}{|c|}{ Totales }} \\
\hline & \multicolumn{2}{|c|}{ CAEF } & \multicolumn{2}{|c|}{ CAEC } & \multicolumn{2}{|c|}{ CAC } & & \\
\hline & 2009 & 2019 & 2009 & 2019 & 2009 & 2019 & 2009 & 2019 \\
\hline $\begin{array}{c}\text { Ciencias Básicas, } \\
\text { Ingeniería y } \\
\text { Tecnología }\end{array}$ & 3 & 1 & 3 & 7 & o & 2 & 6 & 10 \\
\hline $\begin{array}{c}\text { Ciencias Biológicas y } \\
\text { de la Salud }\end{array}$ & 10 & 1 & 3 & 7 & 2 & 10 & 15 & 18 \\
\hline $\begin{array}{l}\text { Ciencias Sociales y } \\
\text { Administrativas }\end{array}$ & 11 & 5 & 4 & 7 & 1 & 7 & 16 & 19 \\
\hline $\begin{array}{c}\text { Ciencias y } \\
\text { Humanidades }\end{array}$ & 4 & 7 & o & o & o & 3 & 4 & 10 \\
\hline Totales & 28 & 14 & 10 & 21 & 3 & 22 & 41 & 57 \\
\hline
\end{tabular}

Fuente: elaboración propia con datos de la UAT (2010) y PRODEP (2019).

Una explicación del mayor número de CA y del nivel de habilitación que tienen las áreas de Ciencias Biológicas y de la Salud y Ciencias Sociales y Administrativas es porque en ellas se ubican los centros de investigación, que son los pioneros en esta función, cuyos grupos de trabajo se renombraron como CA cuando se inició esta política educativa (Viñas, 2017).

El Programa de Fortalecimiento de la Calidad Educativa 2016-2017 de esta institución, por su parte, resalta que sus CA han tenido una evolución significativa debido a las políticas y estrategias institucionales que se orientaron al fomento del trabajo colegiado, reestructuración y financiamiento. Recalca, por ejemplo, que a través del programa institucional CACyPI se dispuso no conformar más CAEF, pero sí impulsar el avance de los CAEC y mantener el ritmo de trabajo de los CAC (UAT, 2016).

Entre los múltiples factores que pueden explicar el crecimiento de los CA sobresale el CACyPI porque es el único programa que ha establecido esta 
universidad con el propósito de apoyarlos. Tuvo una duración de dos años -2013 y 2014-, no tenía antecedente alguno y tampoco se volvió a replicar en las posteriores administraciones. El objetivo declarado del programa fue

[...] contribuir a desarrollar nuevos proyectos de investigación de calidad, mediante el trabajo colegiado de los PTC a través de las LGAC que cultivan sus CA, para formar recursos humanos, desarrollar productos con impacto en los ámbitos institucional, nacional e internacional y mantener y/o mejorar la capacidad académica y el grado de desarrollo. (UAT, 2014, párr. 4)

El CACyPI estuvo dirigido a los $43 \mathrm{CA}$ existentes en ese periodo. El monto disponible cada año fue de un poco más de 10 millones de pesos distribuidos proporcionalmente de acuerdo con el grado de consolidación en el que se encontraba cada uno de los CA. Los CAEF recibieron anualmente entre 120 ooo y 210 ooo pesos; los CAEC obtuvieron entre 240000 y 300 ooo pesos y, finalmente, a los CAC se les otorgó entre 310 ooo y 380 ooo pesos (UAT, 2014).

En 2013, los 43 CA existentes se distribuían de la siguiente manera: 21 (48.8 \%) estaban en calidad de CAEF, 16 (37.2 \%) como CAEC y 6 (13.9 \%) como CAC. Para 2019 ya contaba con $57 \mathrm{CA}$, lo que muestra un incremento tanto en número como en nivel de consolidación: 14 (24.5 \%) están como CAEF, 21 (36.8 \%) como CAEC y 22 (38.5 \%) como CAC. Sin embargo, al analizar el impacto de este programa institucional (CACyPI), se observa que de los 43 CA que en 2013 recibieron financiamiento, 8 de 21 se mantenían como CAEF. Los CAEC disminuyeron de 16 a 13, mientras que los CAC se incrementaron de 6 a 19. No obstante lo anterior, tres de ellos para 2019 ya habían desaparecido. Durante este periodo surgieron también otros 17 nuevos CA (tabla 7). 
Tabla 7. Comparativo de CA de la UATx en dos periodos

\begin{tabular}{|c|c|c|c|c|}
\hline \multirow{2}{*}{ Ã̃o } & \multicolumn{3}{|c|}{ Categoría } & \multirow{2}{*}{ Total } \\
\hline & CAEF & CAEC & CAC & \\
\hline 2013 & 21 & 16 & 6 & 43 \\
\hline 2019 & 14 & 21 & 22 & 57 \\
\hline $2019^{*}$ & 8 & 13 & 19 & 40 \\
\hline
\end{tabular}

Fuente: Elaboración propia a partir de UAT (2014) y PRODEP (2019).

Nota: 2019* solo señala a los CA que recibieron financiamiento en el 2013.

Cabe advertir la necesidad de hacer un análisis de mayor profundidad para identificar otros factores que pudieran haber influido en los CA que mejoraron su nivel de desarrollo, así como en los que desaparecieron. También conviene subrayar que esta política institucional es complementaria a las acciones que realiza el Prodep, ya que de manera periódica sigue convocando a los CA para que concursen, mediante invitaciones abiertas o cerradas, para la obtención de financiamiento federal para realizar sus investigaciones.

Sin menoscabo de los esfuerzos que institucionalmente ha hecho esta universidad, se destaca que la investigación científica está determinada por la política pública que implementa el Gobierno federal, que estableció desde mediados de los años noventa distintos programas con presupuestos extraordinarios. Esto se evidencia, por ejemplo, cuando los profesores de los centros de investigación se renombraron como CA en razón de que eran quienes tenían los mayores niveles de habilitación y membresía SNI, y de esta manera podrían obtener financiamiento para sus proyectos (Viñas, 2017).

\section{Conclusiones}

Esta investigación muestra, particularmente en esta universidad, los 25 años que el Prodep ha estado vigente. Sus objetivos han impactado en la 
habilitación docente, la formación de investigadores y la conformación de grupos de investigación denominados CA.

Un aspecto que resalta es la habilitación docente, pues se ha invertido; de tener una planta docente compuesta en 1996 principalmente por profesores con estudios de licenciatura, pasó a tener una en 2019 en la que la mayoría de sus docentes contaban con estudios de posgrado.

La conformación de CA como política educativa, para que los profesores se dedicaran también a la investigación y no solo a la docencia, se hace visible en el crecimiento gradual que se observa en estas figuras académicas durante el trayecto de análisis. Aunque cabe destacar que las áreas de las ciencias biológicas y administrativas tienen la prevalencia en comparación con las humanidades y las tecnológicas. Esto puede ser debido a la naturaleza de las mismas disciplinas o al ingreso o aceptación tardía para participar en esta política. Convendría más adelante profundizar este análisis desde las mismas disciplinas.

Entre los factores internos que pueden haber influido en el crecimiento y desarrollo de los CA, se encuentran las iniciativas institucionales de fomento y respaldo a los académicos que participan en estos, como lo ejemplifica el programa CACyPI de esta universidad, es decir, las acciones que implementan y los recursos que destinan las universidades directamente para el impulso de los CA redundan en un crecimiento en cantidad y calidad de la investigación y los investigadores. Este hecho es particularmente relevante, considerando que las universidades han tenido la docencia como función central de su actividad educativa, y por tanto, la investigación requiere un número mayor de políticas específicas constantes y duraderas para su consolidación.

Sobresale en esta universidad la inexistencia de la categoría de investigadores en su normatividad, aun cuando un número significativo de 
sus académicos desarrollan esta actividad y han obtenido el reconocimiento por el SNI. Esto significa que la investigación se encuadra, a pesar de lo anterior, solo como una de las funciones del docente de nivel superior, como lo señala el Prodep, y no como una actividad sustantiva de la institución.

Las políticas educativas orientadas al desarrollo de los CA son, sin duda alguna, elementos importantes en el propósito de fortalecer la investigación y los investigadores en las IES. Las estrategias establecidas por el Prodep han incrementado el interés por la investigación científica. Sin embargo, sería conveniente que adicionalmente las universidades implementaran políticas de reconocimiento y mejoramiento de las condiciones laborales de quienes se dedican a la investigación para consolidar su planta de investigadores.

En suma, el Prodep en el largo proceso de aprendizaje y asentamiento ha generado entre los actores educativos - autoridades universitarias y académicos - un entorno institucional de apoyo, reconocimiento y estímulos a favor de la investigación, y ha abierto el camino a los profesores para que, con mayores niveles de habilitación, puedan desarrollar esta importante función de la educación superior.

\section{Sobre el autor}

Felipe Hernández Hernández. Doctor en Ciencias Administrativas. Maestro en Administración Educativa. Licenciado en Lingüística Aplicada por la Universidad Autónoma de Tlaxcala. Licenciado en Educación por la Universidad Pedagógica Nacional de México. Profesor investigador de la Facultad de Ciencias de la Educación de la Universidad Autónoma de Tlaxcala. Líder del Cuerpo Académico Consolidado Gestión y Políticas Educativas. Áreas de interés: políticas educativas, gestión y evaluación institucional y formación docente y directiva. Entre sus publicaciones 
recientes se encuentran los libros Docencia, dirección y gestión, los retos de las instituciones educativas (2019) e Implicaciones de la política educativa en educación superior (2018). Cuenta con el Reconocimiento de Perfil Deseable Prodep-SEP. Es miembro del Sistema Nacional de Investigadores nivel I, socio del Consejo Mexicano de Investigación Educativa (Comie), de la Sociedad Mexicana de Educación Comparada (Somec) y del Círculo Mexicano de Profesores de Filosofía.

\section{Referencias}

Acosta, A. (2006). Señales cruzadas: una interpretación sobre las políticas de formación de cuerpos académicos en México. Revista de la Educación Superior, 35(3), 81-92. http://www.redalyc.org/articulo.oa?id=60413905

Avendaño, W., Paz, L. y Rueda, G. (2017). Políticas públicas y educación superior: análisis conceptual del contexto colombiano. Revista Venezolana de Gerencia, 22(79), 467-485. DOI: https://doi.org/10.37960/revista.v22i79.23034

Becerril, M., Nosnik, A., Laguna, A. y Archundia, E. (2019). Aproximación sistemática de los factores que influyen en la consolidación de los cuerpos académicos en universidades públicas mexicanas. Revista del Centro de Investigación y Estudios Gerenciales (CIEG), (36), 213-226.

Brambila, R., Carrasco, A. C. y Kent, R. (2016). Investigadores de electrónica mexicanos que colaboran para publicar. Coautores que trabajan en red. BiD: Textos Universitaris de Biblioteconomia i Documentació, (37). http://bid.ub.edu/es/37/brambila.htm

Cabrera, J. C. y Díaz-Ordaz, E. M. (2013). Construyendo una identidad colectiva. En J. Cabrera y L. Pons (Coords.), Configuraciones narrativas de grupos y cuerpos académicos en el campo de la investigación educativa (pp. 67-88). Octaedro.

Cardozo, M. (2013). Políticas públicas: los debates de su análisis y evaluación. Andamios, $10(21), 39-59$.

http://www.scielo.org.mx/scielo.php?script=sci $\operatorname{arttext\& pid=S1870-}$ 00632013000100003 
Clark, R. (1998). Crecimiento sustantivo y organización innovadora: nuevas categorías para la investigación en educación superior. Perfiles Educativos, 2o(81), 1-16.

Daft, R. (2004). Administración. Thomson Editores.

Díaz-Barriga, A. (2005). El docente y los programas escolares: lo institucional y lo didáctico. Ediciones Pomares.

Diario Oficial de la Federación. (2010). Reglas de Operación del Programa de Mejoramiento del Profesorado. México. http://promep.sep.gob.mx/reglas\%5CReglas

Diario Oficial de la Federación. (2013). Acuerdo número 712 por el que se emiten las Reglas de Operación del Programa para el Desarrollo Profesional Docente. México: Poder Ejecutivo. Secretaría de Educación Pública.

Diario Oficial de la Federación. (2016). Acuerdo número 21/12/16 por el que se emiten las Reglas de Operación del Programa para el Desarrollo Profesional Docente para el Ejercicio Fiscal 2017. México: Poder Ejecutivo. Secretaría de Educación Pública.

Edel-Navarro, R., Ferra-Torres, G., y De Vries, W. (2018). El Prodep en las escuelas normales mexicanas: efectos y prospectiva. Revista de la Educación Superior, 47(187), 71-92. http://www.scielo.org.mx/scielo.php?script=sci abstract\&pid=S0185$\underline{27602018000300071 \& \operatorname{lng}=\text { es\&nrm=iso\&tlng }=\text { es }}$

Fernández, F. (2002). El análisis de contenido como ayuda metodológica para la investigación. Ciencias Sociales, (96), 35-53.

https://www.revistacienciassociales.ucr.ac.cr/images/revistas/RCS96/03.pdf

Franco, J. (2017). Diseño de políticas públicas: una guía práctica para transformar ideas en proyectos viables. IEXE Editorial.

García, C. (2019). Efectos de las políticas en educación superior en México: mirar las prácticas académicas y educativas. En M. de Sierra y Z. Navarrete (Coords.), Políticas y prácticas educativas en perspectiva comparada (pp. 111-132) UPN.

Gibson, J., Ivancevich, J., Donnelly, J. y Konopaske, R. (2006). Organizaciones. Comportamiento, estructura, procesos. McGraw-Hill Interamericana.

Hargreaves, A. (1996). Colaboración y colegialidad artificial (¿copa reconfortante o cáliz envenenado?). Morata.

Hallazgos

ISSN: 1794-3841 | e-ISSN: 2422-409X | DOI: https://doi.org/10.15332/2422409X

Vol. 18 N.o 36| julio-diciembre del 2021 
Jones, G. (2008). Teoría organizacional. Diseño y cambio en las organizaciones. Pearson Educación.

Jornet, J. M., Carmona, C. y Bakieva, M. (2012). Hacia una definición del constructo de colegialidad docente: estrategias metodológicas para su evaluación. Revista Iberoamericana de Evaluación Educativa (RIEE), 5(1), 179-185. http://www.rinace.net/riee/numeros/vol5-num1_e/art12.html

Kast, E. y Rosenzweing, E. (2004). Administración en las organizaciones: Enfoque de sistemas y de contingencias. McGraw-Hill.

Lobato, O. y Garza, E. (2009). La organización del cuerpo académico: las premisas de decisión, colegialidad y respuesta grupal. Revista Mexicana de Investigación Educativa, 14 (40), 191-216. http://www.redalyc.org/articulo.oa?id=14004010

Magaña, L. (2016). Profesión académica y estímulos económicos en las universidades públicas. Causas institucionales y efectos organizacionales. Reencuentro. Análisis de Problemas Universitarios, 28(71), 33-48. https://reencuentro.xoc.uam.mx/index,php/reencuentro/article/view/893

Maldonado, A. (2005). Comunidades epistémicas: una propuesta para estudiar el papel de los expertos en la definición de políticas en educación superior en México. Revista de la Educación Superior, 34(134), 107-122.

Olivé, L. (2008). La ciencia y la tecnología en la sociedad del conocimiento. Ética, política y epistemología. Fondo de Cultura Económica.

Pérez, O. y Bañuelos, M. (2017). Contexto de docentes investigadores en la Universidad Autónoma de Zacatecas miembros del SNI y del Prodep. Revista Estudios Gerenciales y de las Organizaciones, 1O(3), 217-237.

Programa para el Desarrollo Profesional Docente. (2019). Número de CA reconocidos por PRODEP por grado de consolidación por DES. https://promep.sep.gob.mx/registroca/Reportes-PIFI.php

Revuelta, B. (2007). La implementación de políticas públicas. Dikaion, 21(16), 135-156.

Rueda, M. (2003). Presentación de la colección. La investigación Educativa en México. http://www.comie.org.mx/v1/sitio/portal.php?sec=SC03\&sub=SBB\&opc=OPC01 
Secretaría de Educación Pública. (2006). Programa de Mejoramiento del Profesorado PROMEP. Un primer análisis de su operación e impactos en el proceso de fortalecimiento académico de las universidades públicas. México.

Suárez-Amaya, W., Díaz-Barrios, J., y Pereira, M. (2021). Perfil competitivo como herramienta para la gestión estratégica de la investigación en universidades. Hallazgos, 18(35), 259-286. DOI: https://doi.org/10.15332/2422409X.5768

Universidad Autónoma de Tlaxcala. (1996). La UAT en cifras. Secretaría Técnica.

Universidad Autónoma de Tlaxcala. (2006). Perspectiva universitaria. Contenidos estadísticos de la universidad performativa. Colección Estadística. Departamento de Diseño y Análisis Estadístico-Secretaría Técnica.

Universidad Autónoma de Tlaxcala. (2010). Análisis situacional. Objetivos, políticas específicas y metas. CA de la UATx. Informe Técnico y Guía Operativa. Coordinación General de Cuerpos Académicos.

Universidad Autónoma de Tlaxcala. (2014). Programa estratégico institucional. Cuerpos académicos, calidad y productividad para su internacionalización. Lineamientos generales. Coordinación Institucional de Cuerpos Académicos.

Universidad Autónoma de Tlaxcala. (2016). Programa de Fortalecimiento de la Calidad Educativa 2016-2017. México.

Universidad Autónoma de Tlaxcala. (2019a). Plantilla de personal académico de la UAT. Período primavera 2019. Unidad de Personal Académico.

Universidad Autónoma de Tlaxcala. (2019b). Miembros del Sistema Nacional de Investigadores de la UAT. Secretaría de Investigación Científica y Posgrado.

Viñas, S. (2017). 40 años en la producción de conocimientos por investigación científica. Universidad Autónoma de Tlaxcala. México.

Zúñiga, M., Pérez C., y García, M. (2016). Retos de los académicos en la producción de conocimiento en las universidades públicas estatales de México. Revista Electrónica Pesquiseduca, 8(16), 295-315. 